\section{Schmerztherapie: Ein Update}

\author{
Birgit Kröner-Herwig1
}

Abteilung für Klinische Psychologie und Psychotherapie, Universität Göttingen

psychoneuro 2005; 31 (2): 96-102

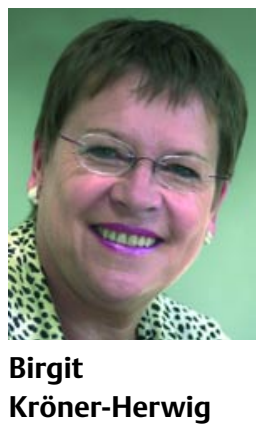

Kröner-Herwig
Verschiedene Metaanalysen erbringen die unabweisbare Evidenz, dass psychologische Schmerztherapie wirksam ist. Diese Aussagen lassen sich wesentlich auf chronische unspezifische Rückenschmerzen, Kopfschmerz vom Typ Migräne und vom Spannungstyp beziehen. Eine der Metaanalysen erweitert diese Syndrompalette, indem sie weitere Schmerzstörungen einbezieht. Das Spektrum der Behandlungsformate umfasst im Wesentlichen kognitiv-behaviorale Verfahren, Relaxation und Biofeedback sowie in begrenztem Ausmaß interdisziplinäre Therapieformate. Andere Therapieverfahren wie die Hypnose und tiefenpsychologisch orientierte Therapie sind bei chronischen Schmerzen sehr selten und fast nie in RCTs eingesetzt worden, so dass sie bei der hier vorgenommenen Analyse keine Beachtung finden konnten. Mit Ausnahme der Kopfschmerzbehandlung bei Kindern und Jugendlichen, bei der die Wirksamkeit, insbesondere die langfristige Wirksamkeit, hoch ist, sind die Effekte der Therapie in verschiedenen Variablen, die Therapieerfolg repräsentieren, von geringer bis mittlerer Größe. Dies bedeutet allerdings auch, dass Psychotherapie bei chronischen Schmerzen weniger wirksam ist als bei genuin psychischen Störungen wie z.B. Angststörungen. Die Wirksamkeit ist aber auch nicht geringer als bei klassischen Verfahren der Schmerztherapie wie der Gabe von Opiaten oder Antidepressiva.
$\mathrm{P}$ sychologische Schmerztherapie entwickelte sich gleichzeitig mit der Konzeptbildung zum „Chronischen Schmerz“, einem Krankheitskonzept, in dem Gemeinsamkeiten der Schmerzstörung jenseits der Unterschiede diverser Syndrome wie Kopfschmerz, Rückenschmerz oder Fibromyalgie hervorgehoben werden. Gemeinsame Merkmale des Chronischen Schmerzes sind nicht nur die lange Dauer der Schmerzbeschwerden und die unzureichende Behandelbarkeit durch konventionelle, medizinische Methoden, sondern auch die Bedeutung psychologischer Faktoren für das Syndrom. So erwies eine große
Zahl von Studien, dass eine hohe Beeinträchtigung des Patienten als die eigentliche Indikation für Schmerztherapie, nicht wesentlich durch das Ausmaß diagnostizierbarer Schädigungen und nur unzureichend durch die erlebte Schmerzintensität oder andere Parameter der Schmerzwahrnehmung bestimmt wird, sondern maßgeblich durch psychologische Prozesse $(10,17)$. Somit liegt die potentielle Nützlichkeit psychologischer Interventionen für die Behandlung chronischer Schmerzsyndrome unmittelbar auf der Hand, nämlich der Versuch, die auf die Beeinträchti- gung Einfluss nehmenden Parameter zu modifizieren (18).

Zu Beginn der Geschichte der psychologischen Schmerztherapie standen allerdings genuine Parameter des Schmerzerlebens (z.B. die subjektive Schmerzintensität) im Fokus des Veränderungsbemühens. Unter der Annahme, dass Stress, Anspannung bzw. ein hohes Aktivierungsniveau schmerzverstärkenden und belastungsverstärkenden Einfluss haben, sind frühzeitig (etwa seit 1974) Relaxationsverfahren in der Schmerztherapie eingesetzt worden. Eine Analyse der angloamerikanischen Datenbanken (PsychInfo) in Fünfjahreszeiträumen (1974-2002) zeigt, dass die Forschung zu Relaxationsverfahren in der Schmerztherapie über die Jahre hinweg einen relativ konstanten Anteil hatte (Min. 1974-1978: 16\%; Max. 1983-1987: 23\%). Der Einsatz von Biofeedbackverfahren als Hauptstrategie der Behandlung mit ähnlichen Zielen wie beim Einsatz von Relaxationsverfahren (Ausnahme Vasokonstriktionstraining bei Migräne) hat über die Jahre hinweg deutlich abgenommen (1978-1982: 27\%; 1992-1997: 13\%). Biofeedback wird heute hauptsächlich als Modul innerhalb multimodaler Behandlungspro-

1 Teile dieses Artikels wurden bereits in „Psychotherapie im Dialog“ (2005) veröffentlicht. 


\begin{tabular}{|c|c|}
\hline $\begin{array}{l}\text { Tab. } 1 \text { Evic } \\
\text { (AV }\end{array}$ & $\begin{array}{l}\text { Izgrade für Klinische Studien } \\
\text { F \& ÄZQ, } 2001^{*} \text { ) }\end{array}$ \\
\hline Evidenzgrad & Evidenzbasis \\
\hline la & Metaanalyse(n) über randomisierte, kontrollierte Studien \\
\hline Ib & mind. 1 randomisierte, kontrollierte Studie(n) (RCT) \\
\hline Ila & $\begin{array}{l}\text { mind. } 1 \text { gut angelegte quasi-experimentelle Studie(n) } \\
\text { (effectiveness, Fallkontrollstudie) }\end{array}$ \\
\hline III & $\begin{array}{l}\text { gut angelegte nicht-experimentelle oder deskriptive Studien } \\
\text { (1 Gruppen-Prä-Post-Vergleich, Korrelationsstudien) }\end{array}$ \\
\hline IV & $\begin{array}{l}\text { unsystematische Einzelfallstudien, Kasuistiken, Meinungen } \\
\text { von Experten, Konsensuskonferenzen, klinische Erfahrung an- } \\
\text { erkannter Autoritäten }\end{array}$ \\
\hline
\end{tabular}

gramme eingesetzt. Von Anfang an hat die Untersuchung behavioraler oder kognitiv-behavioraler Behandlungsstrategien einen hohen Anteil an der gesamten Therapieforschung gehabt, der sich im Laufe der Jahre noch verstärkt hat (1978-1982: 22\%, 1988-1992: 34\%). Diese Verfahren haben ein deutlich breiteres Therapiezielspektrum als Relaxation und Biofeedback.

Einen deutlichen Anstieg im Forschungsspektrum zeigen multidisziplinäre Behandlungsansätze, in die psychologische Interventionen eingebettet sind (1974-1977: 5\%; 1993-1997: 20\%). Psychodynamische Therapieansätze spielen kaum eine Rolle in der Schmerztherapie (Forschungsanteil 1974-1977: 4\%; 1983-1987: 1\%). Der Anteil anderer, den genannten Kategorien nicht zuordenbarer heterogener Verfahren (wie Hypnose, Musiktherapie etc.) liegt zwischen 25\% (1974-1977) und 15\% (1988-1997).

Interessant ist auch die Entwicklung bezüglich der Schmerzsyndrome, deren Behandlung im vorrangigen Interesse der Forschung stand. Während in der frühen Forschung (1974-1977) eindeutig der Kopfschmerz im Zentrum steht (Anteil $48 \%$ ) nimmt das psychologische therapiebezogene Forschungsinteresse im Lauf der Zeit kontinuierlich auf 27\% ab (1993-1997). Der „undifferenzierte“ Chronische Schmerz als Diagnose- und Behandlungskategorie hat seinen Gipfel zwischen 1978 und 1982, bleibt aber bis 1997 mit über 30\% Anteil insgesamt der am häufigsten behandelte „Gegenstand“. Der Rückenschmerz als Einzelsyndrom zeigt über die Jahre einen Zuwachs (8\%-15\%) in der Behandlungsforschung; dies ist in der deutschsprachigen Therapieforschung besonders eklatant (1982-87: 6\%; 1993-1997: $20 \%)$.

Insgesamt fällt auf, dass über die Jahre hinweg neben der Sammelkategorie „Chronischer Schmerz“ der Anteil der behandelten spezifischen chronischen Syndrome außer Kopfund Rückenschmerz deutlich zunimmt (1974-1977: 12\%; 1993-1997: 27\%). Hier können Fibromyalgie, Temperomandibuläre Dysfunktion oder Arthritis genannt werden.

Insgesamt zeigte die psychologische Schmerztherapieforschung die größte Präsenz in der englischsprachigen Literatur in den Jahren 1983-1987, in Deutschland erst 1988-1992. Danach sinkt die Gesamtzahl der Forschungsstudien zur Schmerztherapie eklatant. Im Zeitraum 1988 bis 2002 reduziert sich die Zahl von zuvor ca. 100 veröffentlichten Therapiestudien in der englischsprachigen Literatur innerhalb von fünf Jahren auf weniger als die Hälfte. In der deutschen psychologischen Literatur sind nur fünf empirische Therapiestudien in diesem Zeitraum identifizierbar (Psyndex).

Was bedeutet diese Entwicklung? Wissen wir genug über Wirksamkeit und Mechanismen der psychologischen Schmerztherapie? Ist das Thema "Schmerz" aus dem Interesse der psychologischen Forscher verschwunden, weil sie frustriert sind über die bisherigen Ergebnisse? Um der Beantwortung dieser Frage näher zu kommen, wird im Folgenden dem Stand der
Wirksamkeitsforschung nachgegangen.

\section{Die Wirksamkeit psycho- logischer Schmerztherapie}

Wir folgen bei der Wirksamkeitsbetrachtung den Argumenten der „evidence based medicine“ (26) und beziehen uns im Wesentlichen auf empirische Studien mit der höchsten Validität, nämlich sog. Randomized Controlled Trials (RCTs) (vgl. Tab. 1). Der Stand der Evidenzbasierung psychologischer Schmerztherapie soll anhand von Metaanalysen über RCTs untersucht werden.

\section{Verhaltenstherapie}

Morley et al. (21) veröffentlichten 1999 ein systematisches Review zur kognitiv-behavioralen Therapie bzw. Verhaltenstherapie inkl. Biofeedback bei erwachsenen Patienten mit Chronischem Schmerz. Die Autoren schlossen dabei nur Studien zur Kopfschmerztherapie aus. Nach der Analyse von 30 relevanten Untersuchungen konnten 25 für die Metaanalyse genutzt werden, in die die Daten von ca. 1600 Patienten eingingen. Die berücksichtigten Studien stammen aus den Jahren 1982 bis 1996, wobei sich nur eine Untersuchung deutscher Forscher darunter befindet. Die behandelten Patienten litten unter diversen Schmerzproblemen. Die am häufigsten untersuchte Therapieform war die kognitiv-behaviorale Behandlung. Die Analysen bezogen sich auf verschiedene Wirksamkeitsbereiche, zu denen in den meisten Studien Daten erhoben worden waren (Tab. 2). Die Messung der Therapiezielerreichung erfolgt in der Regel multidimensional und multimethodal (18).

Der Vergleich der psychologischen Behandlungsverfahren mit Wartelistenkontrollgruppen (mit self-monitoring) erbrachte kleine $(\geq 0,36)$ bis mittelgroße Effektstärken $(\leq 0,60)$. Die berechneten Konfidenzintervalle schließen in keinem Fall die 0 ein. Die Effektstärken liegen somit in allen Zielbereichen signifikant über 0 . Die kognitiv-behaviorale Therapie als Behandlung, die in den meisten Studien untersucht wurde, schneidet mit einer Ausnahme in allen Vergleichen bezüg- 
Tab. 2 Chronischer Schmerz: Effektstärken (nach 21)

\begin{tabular}{lllll} 
Bereich & Gesamt & & & \\
\hline & $\mathbf{n}$ & $\begin{array}{l}\text { mittl. Effekt- } \\
\text { stärke }\end{array}$ & $\begin{array}{l}\text { Konfidenz- } \\
\text { intervall 95\% }\end{array}$ & z \\
\hline Schmerzerleben & & 0,40 & $0,22-0,58$ & 4,28 \\
\hline Stimmung/Emotionalität/Depression & 28 & 0,36 & $0,13-0,59$ & 3,11 \\
Stimmung/Emotionalität ohne Depression & 24 & 0,52 & $0,19-0,84$ & 3,10 \\
\hline Coping (negativ) & 16 & 0,50 & $0,27-0,73$ & 4,20 \\
\hline Coping (positiv) & 16 & 0,53 & $0,28-0,78$ & 4,20 \\
\hline Verhaltensausdruck & 11 & 0,50 & $0,22-0,78$ & 3,49 \\
\hline Verhaltensaktivität & 12 & 0,46 & $0,25-0,72$ & 4,34 \\
\hline Interferenz mit sozialer Rolle & 14 & 0,60 & $0,44-0,76$ & 7,28 \\
\hline$\varnothing$ & 25 & 0,50 & & \\
\end{tabular}

lich der Therapiezielerreichung besser ab als die Wartelistenbedingung. Die Autoren berechneten eine weitere Analyse, in der sie sämtliche psychologischen Therapien mit aktiven Vergleichsbedingungen in Beziehung setzten (wie z.B. „routine care“, medizinische Therapie, Physiotherapie), in die insgesamt allerdings weniger Originalstudien als zuvor eingingen. Wenn man die aktiven Vergleichsbehandlungen in die Effektstärkenberechnung einbezieht, werden die Werte deutlich kleiner $(0,05-0,40)$, und nur noch in drei der acht Bereiche ist die Effektstärke signifikant, d.h. aber dennoch dass die kognitiv behaviorale Therapie erfolgreicher ist als der Durchschnitt anderer Behandlungen.

Van Tulder et al. (32) erstellten eine Metaanalyse über die psychologische Behandlung bei unspezifischem chronischen Rückenschmerz für die Cochrane Collaboration (www.cochrane.org), deren Ziel es ist, aktuelle Informationen über die Wirksamkeit von Maßnahmen der Gesundheitsversorgung weltweit und schnell verfügbar zu machen. Sie lässt dafür unter striktesten methodischen Anforderungen systematische Reviews zur Wirksamkeit erarbeiten und dokumentiert diese in ihrer Library. Rückenschmerzen stellen bekanntermaßen das „teuerste" Schmerzsyndrom im Versorgungssystem dar (14). Verwendbarbar für die Metaanalyse waren 20 RCTs aus den Jahren 1982 bis 1997, in die die Daten von ca. 1300 Patienten eingingen. Eine deutsche Studie wurde eingeschlossen. In den Originaluntersuchungen wurden als
Behandlungsverfahren Verhaltenstherapie sowie Relaxation und Biofeedback eingesetzt. Es waren sehr verschiedene Kontrollgruppentypen vertreten, von „no treatment“ über Wartelistenkontrollgruppe, Plazebobehandlung bis hin zu verhaltenstherapeutisch fundierten Alternativbehandlungen. Die Autoren diskutieren verschiedene metaanalytische Vergleiche zwischen psychologischen Behandlungsverfahren (z.B. kognitiv-behaviorale Therapie vs. operante Therapie). Aufgrund der geringen Zahl der Studien, die sie nutzen konnten, ist aber nur die Wirksamkeitsüberprüfung von Verhaltenstherapie gegenüber den Kontrollbedingungen „no treatment“, „Wartelistenkontrolle“ und „Plazebo“ möglich, in die elf Studien einbezogen werden konnten. Die Autoren bildeten drei Therapiezielbereiche: Schmerzintensität, funktioneller Status und Schmerzverhalten (Tab. 2). Alle Effektmaße reflektieren eine kleine bis mittlere Effektivität. Bei der Outcomevariable „funktioneller Status“ wird im Konfidenzbereich ein Wert von nahezu 0 eingeschlossen. Die insgesamt großen Konfidenzintervalle weisen auf die Heterogenität der Ergebnisse hin, die bei der Erfolgsvariablen „Schmerzintensität“ allerdings bis in den hohen Wirksamkeitsbereich reichen $(0,98)$. Wenn der Vergleich nur mit „aktiven“ Kontrollgruppen durchgeführt wird, reduzieren sich die Effektstärken weiter (0,03-0,31).

Die Autoren kommen zu dem Schluss, dass Verhaltenstherapie auf eine deutliche empirische Evidenz verweisen kann (Stufe Ia). Über die relative Wirksamkeit verschiedener psychologischer Therapieverfahren lassen sich auf der Basis der Metaanalyse keine reliablen Aussagen machen.

\section{Multidisziplinäre Reha- bilitation bei chronischen Rückenschmerzen}

Eine weitere Analyse der Cochrane Bibliothek legten Guzmán et al. (9) vor, in der sie die Wirksamkeit multidisziplinärer Rehabilitation bei chronischen Rückenschmerzen (ca. 1900 Patienten) untersuchten. Psychologische Interventionen sind regelhaft Bestandteil dieser Programme (28). Unter den meisten Schmerzexperten im Bereich der Therapie und Rehabilitation besteht die Annahme, dass diese multidisziplinären Programme das Optimum der aktuellen Schmerzbehandlung darstellen (18). In den Studien, die zwischen 1989 und 1997 veröffentlicht wurden, darunter eine deutsche Untersuchung, wurden Patienten mit Rückenschmerz von mindestens dreimonatiger Dauer und mit Beeinträchtigung der Arbeitsfähigkeit oder der alltäglichen Lebensvollzüge eingeschlossen. Als multidisziplinäre Behandlung wurde ein Programm gewertet, das ein körperliches Trainingssmodul enthielt und ein Modul, das entweder aus psychologischer Beratung bzw. Therapie oder aus einer sozialen bzw. berufsbezogenen Intervention bestand. Als Kontrollbedingung galt jede Behandlung, die diesen Kriterien nicht entsprach. Reine Rückenschulen (Gruppenedukation und Skill-Training) wurden nicht einbezogen. Die zehn Studien, 
die die Kriterien der Autoren erfüllten, wurden einzeln hinsichtlich des Erfolgs oder Misserfolgs des multidisziplinären Settings in den einzelnen Therapieerfolgsvariablen beurteilt. Die Autoren kommen zu dem Schluss, dass es deutliche Hinweise dafür gibt, dass eine umfangreiche multidisziplinäre rehabilitative Behandlung (> 100 Stunden) erfolgreicher ist als eine nicht-multidisziplinäre, wenn man den funktionellen Status der Patienten betrachtet und abgeschwächt, wenn Schmerzerleben als Zielvariable herangezogen wird. Bei einer weniger umfangreichen Behandlung besteht ein Wirksamkeitsnachweis nach Meinung der Autoren nicht. Nicht klären lässt sich über diese Analyse der Stellenwert psychologischer Anteile in der Behandlung. Insgesamt ist die von Guzmán et al. vorgestellte Analyse (9) nicht in allen Details überzeugend bzw. nachzuvollziehen.

\section{Kopfschmerzbehandlung}

Die beiden im Folgenden vorgestellten Reviews befassen sich mit der Behandlung von primärem Kopfschmerz, nämlich der Migräne und dem Kopfschmerz vom Spannungstyp (KST). Diese beiden Metaanalysen werden von Holroyd (12) in seinem Überblicksartikel zur Erfassung und Behandlung von chronischem Kopfschmerz vorstellt. Beide Metaanalysen wurden für die amerikanische Agency for Health Care Policy and Research angefertigt $(8,20)$, deren Anforderungen an eine solche Analyse denen der Cochrane Collaboration vergleichbar sind. Immerhin 39 RCTs konnten Goslin et al. (8) für ihre Metaanalyse zur psychologischen Behandlung von Migräne identifizieren. Die Forschungsstudien evaluierten verschiedene Behandlungsverfahren (Relaxation, Temperatur-Biofeedback, EMG-Biofeedback, kognitive Verhaltenstherapie und Kombinationen), für die getrennt globale Effektstärken ausgewiesen werden. Die Autoren beschreiben Effektstärken (hier Intragruppen-Effektstärken, die Veränderungen zwischen dem Zeitpunkt vor und nach der Therapie kennzeichnen). Anders als in den Reviews zuvor werden hier reine Schmerzparameter (Häufigkeit, Dauer, Intensität) als OutcomeVariablen verwendet.

Es finden sich Effektstärken von $\mathrm{d}=0,37$ bis $\mathrm{d}=0,77$, während die Wartelistenkontrollgruppen im Durchschnitt ein d von 0 erreichen und die Plazebobehandlungen ein d von 0,16 . Nur der Vergleich von Hauttemperaturfeedback und einer Kombination von Temperatur-Biofeedback mit anderen Verfahren (insgesamt acht Studien) zeigt keine signifikante Überlegenheit gegenüber der Wartelistenbedingung. Insgesamt bedeutet dies, dass die psychologisch behandelten Migränepatienten eine deutlich größere Veränderung in der Kopfschmerzaktivität zeigen als auf die Behandlung wartende Patienten oder Patienten mit unspezifischer Intervention.

McCrory et al. (20) analysieren Behandlungsstudien, bei denen es um die Therapie des KST geht. Sie identifizieren 35 verwertbare Originalstudien. Bei Holroyd (12) findet sich eine Graphik zu den globalen Effektstärken dieser Metaanalyse bezogen auf kognitive Verhaltenstherapie, Relaxation, EMG- und Handtemperatur-Biofeedback sowie Kombinationen dieser Verfahren. Auch hier liegen Effektstärken in ähnlichen Bereichen wie sie in der MigräneMetaanalyse gefunden wurden $(\mathrm{d}=$ 0,4-0,80). Auch hier zeigen die Wartegruppen-Kontrollen keinerlei Änderung (Effektstärke um 0). Außer bei einer Kombination von Temperatur-Biofeedback und Relaxation schließen die Konfidenzintervalle den Wert 0 nicht ein. Dies heißt auch im schlechtesten Fall verändert die Therapie die Symptomatik zum Positiven. Betrachtet man die mittlere Reduktion der Kopfschmerzen bezogen auf das Ausgangsniveau durch die verschiedenen psychologischen Behandlungen, so liegt diese bei KST zwischen 40 und 50\% und bei der Migräne nahe $40 \%$.

Diesen Analysen folgend wertet das US Headache Consortium, ein Zusammenschluss verschiedener medizinischer Organisationen, der sich mit der Entwicklung von Guidelines befasst, neuerdings psychologische Behandlungsverfahren (der o.g. Art) als „treatment options for the prevention of migraine“ (12). Dies sollte nach Datenlage auch für den KST gelten.

\section{Kinder und Jugendliche}

Es liegen auch metaanalytische Befunde für Kinder und Jugendliche mit Schmerzen vor. Von 18 Studien (RCTs) konnten Eccleston et al. (5) in ihrer Übersichtsarbeit 13 Studien metaanalytisch aufarbeiten (davon zwei zu Bauchschmerz, eine zur Sichelzellenanämie und der Rest zu Kopfschmerzen). Zu den eingesetzten Behandlungsformen gehören wie bei Erwachsenen Relaxation, Biofeedback, kognitive Verhaltenstherapie und Kombinationen. Die Kontrollbedingungen sind zumeist Wartelistengruppen. Eccleston et al. benutzen für ihre Analyse des Outcomes ein Responderkriterium ( $\geq 50 \%$ Reduktion der Schmerzaktivität) und berechnen damit das Odds Ratio, was in weiten Bereichen das relative „Risiko“ oder, in diesem Fall, die relative Chance beschreibt, im Vergleich zu einem Patienten aus der Kontrollbedingung von der Behandlung zu profitieren. Sie errechnen einen Wert von 8,83 (KI: 4,33-18,03). Damit hat ein behandeltes Kind eine nahezu neunfach größere Chance als ein nicht psychologisch behandeltes Kind, eine deutliche Schmerzverbesserung zu erleben. Als weiteres Maß bestimmten sie die "number needed to treat" (NNT), die Anzahl der Kinder, die behandelt werden müssen, damit ein Erfolg (responder) erzielt wird. Die NNT lag bei $2,32(\mathrm{CI}=1,96-2,88)$. In der eigenen Arbeitsgruppe (15) wurde die Analyse in unterschiedlicher Form und zwar nur zum pädiatrischen Kopfschmerz wiederholt. Von 23 RCTs (veröffentlicht 1984 bis 2002) konnten 15 für zwei Metaanalysen genutzt werden (mit ca. 800 Kindern und Jugendlichen im Alter von 9-22 Jahren). Diese Studien umfassen nur zum Teil die von Eccleston et al. analysierten, da einige neue Studien hinzugekommen sind und andere Studien aus der Analyse herausfielen. Die Auswertung nach dem Effektmaß Hedges' g ergibt bei einer Responder-Analyse den EffektScore von $\mathrm{g}=0,90$ zum Zeitpunkt post (KI: 0,57-1,16). Die dimensio- 
nale Analyse von Kopfschmerzintensität, -dauer und -frequenz zeigt im Intragruppenvergleich prä-post eine mittelhohe Wirksamkeit ( $g=0,60$ ) (KI: 0,31-0,73), im Vergleich prä-follow-up aber eine deutlich größere Effektivität der Therapie in der Höhe von $g=1,0$ (KI: 0,64-1,33), während die Kontrollgruppen im prä-postVergleich nur einen Wert von $\mathrm{g}=0,1$ (KI: -0,26-0,37) erreichen.

\section{Zusammenfassung und kritische Diskussion}

In dem Versuch der Evaluation der Effektivität wurde hier nur auf Metaanalysen von RCTs zurückgegriffen, die die Evidenzbasierung auf der höchsten, d.h. anspruchsvollsten Ebene (1a) ermöglichen. Verschiedene Metaanalysen erbringen die unabweisbare Evidenz, dass psychologische Schmerztherapie wirksam ist. Diese Aussagen lassen sich wesentlich auf chronische unspezifische Rückenschmerzen, Kopfschmerz vom Typ Migräne und vom Spannungstyp beziehen. Eine der Metaanalysen erweitert diese Syndrompalette, indem sie weitere Schmerzstörungen einbezieht. Das Spektrum der Behandlungsformate umfasst im Wesentlichen kognitivbehaviorale Verfahren sowie Relaxation und Biofeedback sowie in begrenztem Ausmaß interdisziplinäre Therapieformate. Andere Therapieverfahren wie die Hypnose und tiefenpsychologisch orientierte Therapie sind bei chronischen Schmerzen sehr selten und fast nie in RCTs eingesetzt worden, so dass sie bei der hier vorgenommenen Analyse keine Beachtung finden konnten. Mit Ausnahme der Kopfschmerzbehandlung bei Kindern und Jugendlichen, bei der die Wirksamkeit, insbesondere die langfristige Wirksamkeit (Follow-up bis ca. 1 Jahr) hoch ist, sind die Effekte der Therapie in verschiedenen Variablen, die Therapieerfolg repräsentieren, von geringer bis mittlerer Größe. Dies bedeutet allerdings auch, dass Psychotherapie bei chronischen Schmerzen weniger wirksam ist als bei genuin psychischen Störungen wie z.B. Angststörungen $(24,25)$.

Wie diese Befunde zum Stand der Evidenzbasierung zu bewerten sind, sollte mit Hilfe des Vergleiches von Analysen zur Wirksamkeit von Verfahren aus dem medizinischen Behandlungsbereich verdeutlicht werden. Obwohl hier keine systematische Analyse vorgenommen werden kann, sollen jedoch einige Eindrücke der Autorin mitgeteilt werden. Bei der Durchsicht der Cochrane Library zum Thema Schmerzbehandlung überrascht die insgesamt relativ geringe Zahl der Reviews zu medizinischen Behandlungsverfahren bei chronischen Schmerzen. Schaut man in einige Reviews hinein, so findet man zumeist eine ähnlich kleine oder kleinere Anzahl von Studien zum evaluierten Behandlungsformat wie bei den Metaanalysen zu den psychologischen Verfahren. In einem Review zur Behandlung von Rückenschmerzen mit Antidepressiva konnten nur neun RCTs ausgewertet werden. Hier wird eine Effektstärke von 0,41 (KI: 0,22-0,61) für die Zielvariable Schmerzstärke angegeben, die in dem Bereich der Wirksamkeit psychologischer Verfahren liegt. Interessanterweise liegt der Effekt für die „Verbesserung der Aktivitäten des täglichen Lebens" als der bedeutsameren Zielvariable nur bei $\mathrm{d}=$ 0,24 . Das Konfidenzintervall schließt sogar negative Grenzwerte ein, d.h. dass die Antidepressiva den Zustand des Patienten auch verschlechtern können (1). Für Rückenmarkstimulation bei chronischem Rückenschmerz wird nur ein RCT berichtet (19). Selbst bei der Anwendung nicht-steroidaler anti-inflammatorischer Pharmaka (NSAID), die als das Mittel der Wahl bei der Therapie von akutem Rückenschmerz gelten, kommen die Autoren zur folgenden Schlussfolgerung: „Sufficient evidence on chronic low back pain is still lacking“ (34). Muskelrelaxantien wird zwar ihre Wirksamkeit für chronische unspezifische Rückenschmerzen bestätigt, auf die Berücksichtigung der signifikant erhöhten unerwünschten Wirkungen dieser Pharmaka wird aber explizit verwiesen (33). Diese sind bei psychologischer Therapie eher nicht zu erwarten. Im Bereich der Massage wird von neun RCTs berichtet, wobei hier Massage aber häufiger mit Eduka- tion und körperlichen Übungen kombiniert war, was Aussagen über die Wirksamkeit von Massage allein unmöglich macht (7). Zu TENS (Transkutane elektrische Nervenstimulation) wird angemerkt, dass die identifizierten 19 RCTs keine Antwort auf einen länger anhaltenden Effekt (über Tage hinaus) der Behandlung ermöglichen (4). Eine Arbeitsgruppe der DGSS hat eine Metaanalyse zur Wirkung von Opiaten bei chronischen, nicht durch Tumore bedingten Schmerzen erarbeitet (27). Das vorläufige Ergebnis ist überraschend. Bei ausschließlicher Berücksichtigung von RCTs ergibt sich eine mittlere Effektstärke von $\mathrm{g}$ $=0,41$, Die ,number needed to treat“ beträgt 4,41 (Pädiatrischer Schmerz: NNT = 2,31, 5). Diese Daten erlauben die vorsichtige Aussage, dass es um die Evidenzbasierung medizinischer Verfahren, auch jener, die besonders häufig eingesetzt werden, nicht zum Besseren steht, so dass in diesem Referenzrahmen die psychologische Therapieforschung bzw. die Wirksamkeit psychologischer Therapie recht gut abschneidet.

Daten zum direkten Vergleich psychologischer Maßnahmen und medizinischer Maßnahmen liegen nur in sehr begrenzter Anzahl vor. Holroyd (12) weist in seinem Artikel zum Stand der Kopfschmerzforschung darauf hin, dass bei der Migräneprävention die prophylaktischen Medikamente Flunarizin und Propranolol ähnliche Therapieerfolge (in \% Verbesserung) erbringen wie Biofeedback. Dies gilt auch für das Antidepressivum Amitriptylin bei KST. Eine frühere Metaanalyse von Hermann et al. (11), in die sowohl psychologische Kopfschmerzbehandlungsverfahren wie pharmakologische Therapien eingingen, zeigt für Kinder und Jugendliche eine mindestens gleich hohe Wirksamkeit, in manchen Fällen sogar eine höhere Wirksamkeit der nichtmedikamentösen Therapieverfahren.

Wenn auch der Stand der Evidenzbasierung der psychologischen Therapie sogar im Vergleich nicht als schlecht zu beurteilen ist, so sind nichtsdestotrotz erhebliche Mängel zu konstatieren. Zum einen ist die 
typische Variante der Kontrollbedingung die Warteliste, die zur Überschätzung des Nutzens spezifischer psychologischer Interventionen Anlass gibt. Somit sollten in Zukunft mehr Vergleichsinterventionen eingeführt werden, die den Effekt der therapeutischen Zuwendung und der subjektiven Erfolgserwartung des Patienten besser kontrollieren können. Auch wären Designs sinnvoll, die den additiven Effekt psychologischer Intervention gegenüber „routine care“ bei Patienten deutlicher bestimmen könnten. Studien-Designs mit Wartelistegruppen haben zumeist auch die Folge, dass keine Intergruppen-Effektstärken für die Follow-up-Untersuchungen bestimmt werden können, da die Patienten der Wartegruppen bereits behandelt werden. Dabei betrifft das Hauptziel von Schmerztherapie natürlich die langfristigen Effekte der Behandlung. Über diese Langzeiteffekte wissen wir deshalb nur ganz wenig (18).

Für die Einschätzung der komparativen Wirksamkeit verschiedener psychologischer Verfahren gibt es zu wenige Studien. Allerdings drängt sich der Eindruck auf, dass keines der Verfahren eine deutliche Überlegenheit gegenüber einem anderen zeigt.

So gut wie nichts wissen wir darüber, welche Therapiemodule der psychologischen Behandlungsverfahren, die ja fast immer als „Packet“ geprüft werden, besonders wirksam sind. Welche Packetbestandteile sind also notwendig, welche nicht? Im Lichte mancher Ergebnisse scheint es, als seien die Grenzen der sinnvollen und nützlichen Addition von Therapiemodulen oft überschritten (30). Somit wäre es u.U. sinnvoller zu fragen, welche psychologischen Interventionen im Rahmen von Programmen eliminiert werden könnten (bei Erhalt der Effektivität), als welche noch hinzugefügt werden sollten, um die Wirksamkeit zu steigern.

Keine endgültigen evidenzbasierten Antworten gibt es auch auf die Frage nach der Indikation für psychologische Schmerztherapie. So wird oft eine hohe subjektive Beeinträchtigung oder das Stadium der
Chronifizierung als Indikation diskutiert (22). Ob die Beachtung des transtheoretischen Modells von Prochaska et al. (23) mit einer Orientierung der Intervention am Stadium der Veränderung des Patienten (Sorglosigkeit, Bewusstwerden, Vorbereitung, Handlung, Aufrechterhaltung) mehr Erfolg in der Therapie nach sich zieht, ist bisher nicht geklärt. Ob die Ergebnisse der amerikanischen Forschung zum besseren Abschneiden der nach dem Multidimensionalen Pain Inventory (vgl. MPI-D, 6) als „dysfunctional“ und „interpersonal distressed“ klassifizierten Patienten in der Therapie auch in Deutschland repliziert werden können $(29,31)$, ist noch nicht überprüft worden. Ob eine stärkere Anpassung der Intervention auf das spezifische Syndrom anstelle der Anwendung eines doch relativ ähnlichen Therapieformat für „Chronischen Schmerz" allgemein zu höherer Wirksamkeit führt, ist eine Frage, auf die es noch keine Antwort gibt.

Die hier als Basis der Diskussion der Wirksamkeit herangezogenen RCTs haben selbstverständlich auch Schwächen, die wesentlich in der möglicherweise eher geringeren externen Validität liegen. Daher sollten auch naturalistische Studien, wie die von Klinger (13) mit der „normalen“ Patientenklientel aus Klinik- oder Praxissetting, häufiger durchgeführt werden. Kontrollierte Einzelfallstudien aus dem Schmerzbereich sind mit einigen Ausnahmen (16) nach Kenntnis der Autorin nicht existent.

Die meisten praktisch tätigen Schmerzmediziner und zum Teil auch Psychologen gehen von einer Optimierung der Therapieergebnisse bei einer Integration von medizinischen und psychologischen Interventionen aus. Dies konnte in eigenen Studien auch verdeutlicht werden $(2,3)$.

Fazit ist damit also, dass die Wirksamkeit psychologischer Schmerztherapie als bestätigt gelten kann, dass aber dennoch viele wichtige Fragen offen sind, so dass dem Trend der Vernachlässigung schmerztherapeutischer Fragestellungen in der Forschung entgegen gewirkt werden musste. Neben den in der Diskussion aufgeworfenen Fragen sollte die For- schung sich auch die Frage stellen, in wie weit der therapeutische Ansatz verbessert werden könnte, um das Ausmaß der Wirksamkeit zu steigern.

Die Schlussfolgerung dieser Ausführungen liegt auf der Hand. Psychologische Schmerztherapie ist ein bedeutsamer Bestandteil des breiten Spektrums schmerztherapeutischer Verfahren. Sie sollte dem Patienten zumindest in allen tertiären schmerztherapeutischen Einrichtungen zur Verfügung stehen. Leider ist dies bisher nur - und auch hier wohl nicht im zufriedenstellenden Ausmaß - in spezialisierten stationären Einrichtungen oder Ambulanzen an Kliniken gewährleistet.

\section{Pain Therapy - An Update} Various meta-analyses provide undeniable evidence that psychological pain treatment is effective. This statement can be applied in the main to chronic, unspecific back pain, migraine and headaches of the tension type. One of the meta-analyses expands this spectrum of syndromes by including further painful disorders. For the most part, the spectrum of treatment formats covers cognitivebehavioural procedures, relaxation and biofeedback, as also, to a limited extent, interdisciplinary therapeutic formats. Other therapeutic measures, such as hypnosis and depth-psychological treatment are rarely applied to chronic pain and are almost never employed in RCTs, so that they have been left out of consideration in the present analysis. With the exception of the treatment of headaches in children and adolescents, which is particularly effective over the long-term (follow-up to approximately 1 year), the effects of treatment in a number of variables reflecting treatment outcome, extend from mild to moderate. This, however, also means that psychotherapy of chronic pain is less effective than of genuine psychic disorders, such as, for example, anxiety disorders. But psychotherapy is at least as effective as classical therapy of pain, e.g opioids and antidepressants.

\section{Key Words}

psychological pain - treatment efficency - metaanalysis - back pain headache - pediatric pain 


\section{Literatur}

1. Assendelft W], Morton SC, Yu EI, Suttorp MJ, Shekelle PG. Spinal manipulative therapy for low back pain. Annals of Internal Medicine 2003; 138: 871-881

2. Basler HD, Jäkle C, Kröner-Herwig B. Cognitive-behavioral therapy for chronic headache at German pain centers. International Journal of Rehabilitation and Health 1996; 2: 235-252

3. Basler HD, Jäkle C, Kröner-Herwig, B. Incorporation of cognitive-behavioral treatment into the medical care of chronic low back patients: a controlled randomized study in German pain treatment centers. Patient Education and Counseling 1997; 31: 113-124

4. Carroll D, Moore RA, McQuay HJ, Fairman F, Tramèr M, Leijon G. Transcutaneous electrical nerve stimulation (TENS) for chronic pain. The Cochrane Database of Systematic Reviews 2000, Issue 4. Art. No.: CD003222. DOI: 10.1002/14651858.CD 003222

5. Eccleston C, Yorke L, Morley S, Williams AC de C, Mastroyannopoulou K. Psychological therapies for the management of chronic and recurrent pain in children and adolescents (Cochrane Review). In: The Cochrane Library, Issue 3. Chichester, UK: John Wiley \& Sons, Ltd, 2004

6. Flor H, Rudy TE, Birbaumer N, Streit B, Schugens MM. Zur Anwendbarkeit des West-Haven-Yale Multidimensional Pain Inventory im Deutschen Sprachraum. Daten zur Reliabilität und Validität des MPI-D. Der Schmerz 1990; 4: 82-87

7. Furlan AD, Brosseau, L, Immanura M, Irvin $E$. Massage for low-back pain (Cochrane Review). In: The Cochrane Library, Issue 3. Chichester, UK: John Wiley \& Sons, Ltd, 2004 8. Goslin RE, Gray RN, Mc Crory DC, Penzien D, Rains J, Hasselblad V. (1999). Behavioral and physical treatments for migraine headache. Technical review 2,2, February 1999. Prepared for the Agency for Health Care Policy and Research under Contract No. 290-94-2025.

9. Guzmán J, Esmail R, Karjalainen K, Malmivaara A, Irvin E, Bombardier C. Multidisciplinary Bio-Psycho-Social Rehabilitation for Chronic Low Back Pain (Cochrane Review). In: The Cochrane Library, Issue 2, 2003. Oxford: Update Software,

10. Hasenbring M, Pfingsten M. Psychologische Mechanismen der Chronifizierung Konsequenzen für die Prävention. In: Basler HD, Franz C, Kröner-Herwig B, Rehfisch HP (Hrsg.). Psychologische Schmerztherapie Heidelberg: Springer, 2004: 99-116

11. Hermann C, Kim M, Blanchard EB. Behavioral and prophylactic pharmacological intervention studies of pediatric migraine: an exploratory metaanalysis. Pain 1995; 60 : 239-256

12. Holroyd KA. Assessment and psychological management of recurrent headache disorders. J Consult Clin Psychol 2002; 70(3): 656-677

13. Klinger R. Evaluation eines stationären Trainings zur Krankheitsbewältigung bei chronischen Rückenschmerzen. Regensburg: S. Roderer Verlag, 1995
14. Kohlmann T, Schmidt CO. Epidemiologie des Rückenschmerzes. In: Hildebrandt J, Müller G, Pfingsten M (Hrsg.). Lendenwirbelsäule - Ursachen, Diagnostik und Therapie von Rückenschmerzen. München: Urban \& Fischer, 2005: 3-13

15. Kremberg E, Lackschewitz H, KrönerHerwig B. Meta-analytische Befunde zur psychologischen Intervention bei pädiatrischem Kopfschmerz. 44. Kongress der Deutschen Gesellschaft für Psychologie (DGPs), Göttingen, September, 2004

16. Kröner-Herwig B, Franz C, Geissner E. (Hrsg.). Praxisfeld Schmerztherapie - Psychologische Behandlung chronischer Schmerzsyndrome. Stuttgart: Thieme, 1999

17. Kröner-Herwig B. Schmerz - eine Gegenstandsbeschreibung. In: Basler HD, Franz C, Kröner-Herwig B, Rehfisch HP. (Hrsg.). Psychologische Schmerztherapie. Heidelberg: Springer, 2004: 3-15

18. Kröner-Herwig B, Frettlöh J. Behandlung chronischer Schmerzsyndrome: Plädoyer für einen multiprofessionellen Therapieansatz. In: Basler HD, Franz C, KrönerHerwig B, Rehfisch HP. (Hrsg.). Psychologische Schmerztherapie Heidelberg: Springer, 2004: 499-522

19. Mailis-Gagnon A, Furlan AD, Sandoval JA, Taylor R. Spinal cord stimulation for chronic pain (Cochrane Review). In: The Chochrane Library, Issue 3. Chichester, UK: John Wiley \& Sons, Ltd, 2004

20. McCrory D, Penzien D, Hasselblad V, Gray R. Behavioral and physical treatments for tension-type and cervicogenic headache. Des Moines, IA: Foundation for Chiropractic Education and Research, 2001

21. Morley S, Eccleston C, Williams A. Systematic review and meta-analysis of randomized controlled trials of cognitive behaviour therapy and behaviour therapy for chronic pain in adults, excluding headache. Pain 1999; 80, 1-13

22. Pfingsten M, Schöps P, Wille T, Terp L, Hildebrandt J. Chronifizierungsausmaß von Schmerzerkrankungen. Quantifizierung. Der Schmerz 2000; 14: 10-17

23. Prochaska JO, DiClemente CC, Norcross JC. In search of how people change. American Psychologist 1992; 47, 1102-1114

24. Ruhmland M, Margraf J. Effektivität psychologischer Therapien von generalisierter Angststörung und sozialer Phobie: MetaAnalysen auf Störungsebene. Verhaltenstherapie 2001; 11(1): 27-40

25. Ruhmland M, Margraf J. Effektivität psychologischer Therapien von Panik und Agoraphobie: Meta-Analysen auf Störungsebene. Verhaltenstherapie 2001; 11(1): 41-53

26. Sackett PL, Sharon S, Richard WS, Rosenberg W, Haynes RB. Evidence-Based Medicine. Edinburgh: Churchill Livingstone, 2000

27. Sorgatz $\mathrm{H}$ et al. Statistische Metaanalyse zur Opioidanwendung bei nichttumorbedingtem Schmerz. Unveröffentlicht. TUDarmstadt, 2002

28. Staal JB, van Tulder MW, Koke AJ, Smid $\mathrm{T}$, van Mechelen W. Return-to-work interventions for low back pain: a descriptive re- view of contents and concepts of working mechanisms. Sports-Medicine 2002; 32, 251-267

29. Strategier LD, Chwalisz K, Altmaier EM, Russell DW, Lehmann TR. Multidimensional assessment of chronic low back pain: Predicting treatment outcomes. Journal of Clinical Psychology in Medical Settings 1997; 4, 91-110

30. ter Kuile MM, Spinhoven P, Linssen ACG, Zitman FG, Van Dyck R, Rooijmans HGM. Autogenic training and cognitive self-hypnosis for the treatment of recurrent headaches in three different subject groups. Pain 1994; 58: 331-340

31. Turk DC, Rudy TE. The robustness of an empirically taxonomy of chronic pain patients. Pain 1990; 43, 27-35

32. van Tulder MW, Ostelo RW, Vlaeyen JW, Linton SJ, Morley SJ, Assendelft WJ. Behavioural treatment for chronic low-back pain (Cochrane Review). In: The Cochrane Library, Issue 3. Chichester, UK: John Wiley \& Sons, Ltd, 2004

33. van Tulder MW, Touray T, Furlan AD, Solway S, Bouter LM. Muscle relaxants for non-specific back pain (Cochrane Review). In: The Cochrane Database of Systematic Reviews 2003, Issue 1. Art. No.: CD004252. DOI: $10.1002 / 14651858 . C D 004252$

34. van Tulder MW, Scholten RJ, Koes BW, Deyo RA. Non-steroidal anti-inflammatory drugs for low-back pain (Cochrane Review). Non-steroidal anti-inflammatory drugs for low-back pain. In: The Cochrane Database of Systematic Reviews 2000, Issue 2. Art. No.: CD000396. DOI: 10.1002/14651858.CD 000396

Korrespondenzadresse:

Prof. Dr. Birgit Kröner-Herwig Abteilung für Klinische Psychologie und Psychotherapie Universität Göttingen

Goßlerstr. 14

BKroener@uni-goettingen.de 